\title{
UN RELIEVE OLMECA EN TENOSIQUE, TABASCO
}

\author{
Roberto García Moll \\ Departamento de Monumentos \\ Prehispánicos del I.N.A.H.
}

En nuestro medio no es raro escuchar expresiones de menosprecio a los trabajos monográficos, o a las simples descripciones de objetos arqueológicos aislados que, por alguna causa, han llegado hasta nosotros. Pero junto con el objeto nos llega gran parte de la información referente a esas piezas, y es importante contar, en un momento dado, con este tipo específico de reportes, ya que no sólo han contribuido al rescate de infinidad de piezas y a la denuncia de saqueos, siendo en gran medida, y lamentablemente, las únicas fuentes de información que tenemos para grandes áreas de Mesoamérica. Consideramos que estas razones son suficientes no sólo para continuar con este tipo de reportes o notas como la que en esta ocasión trataremos, sino que nos atreveríamos a pugnar por su sistemática publicación en revistas especializadas, sobre todo cuando se trata de materiales culturales alejados del área considerada como original.

Con la apertura de nuevas tierras destinadas al cultivo y a la ganadería, así como con el trazo de caminos y las obras de drenaje, en unos casos, y de irrigación en otros, aceleradamente se ha ido transformando la selva tropical de los Estados de Tabasco, Chiapas, Campeche y el Petén Guatemalteco. Desgraciadamente, a partir de estas alteraciones en el medio es que se ha puesto al descubierto la presencia de multitud de restos arqueológicos, que no sólo habían permanecido protegidos por la exhuberante vegetación, sino por la falta de comunicaciones; no obstante, una vez iniciado el proceso de colonización de esta vasta región, han comenzado a destruirse casi simultáneamente.

En esta destrucción irreversible de nuestro pasado cultural no sólo intervienen los factores de progreso, sino también, en un alto grado, la comercialización de una mínima parte de los objetos que los saqueadores extraen de los sitios arqueológicos. De esta manera, des- 


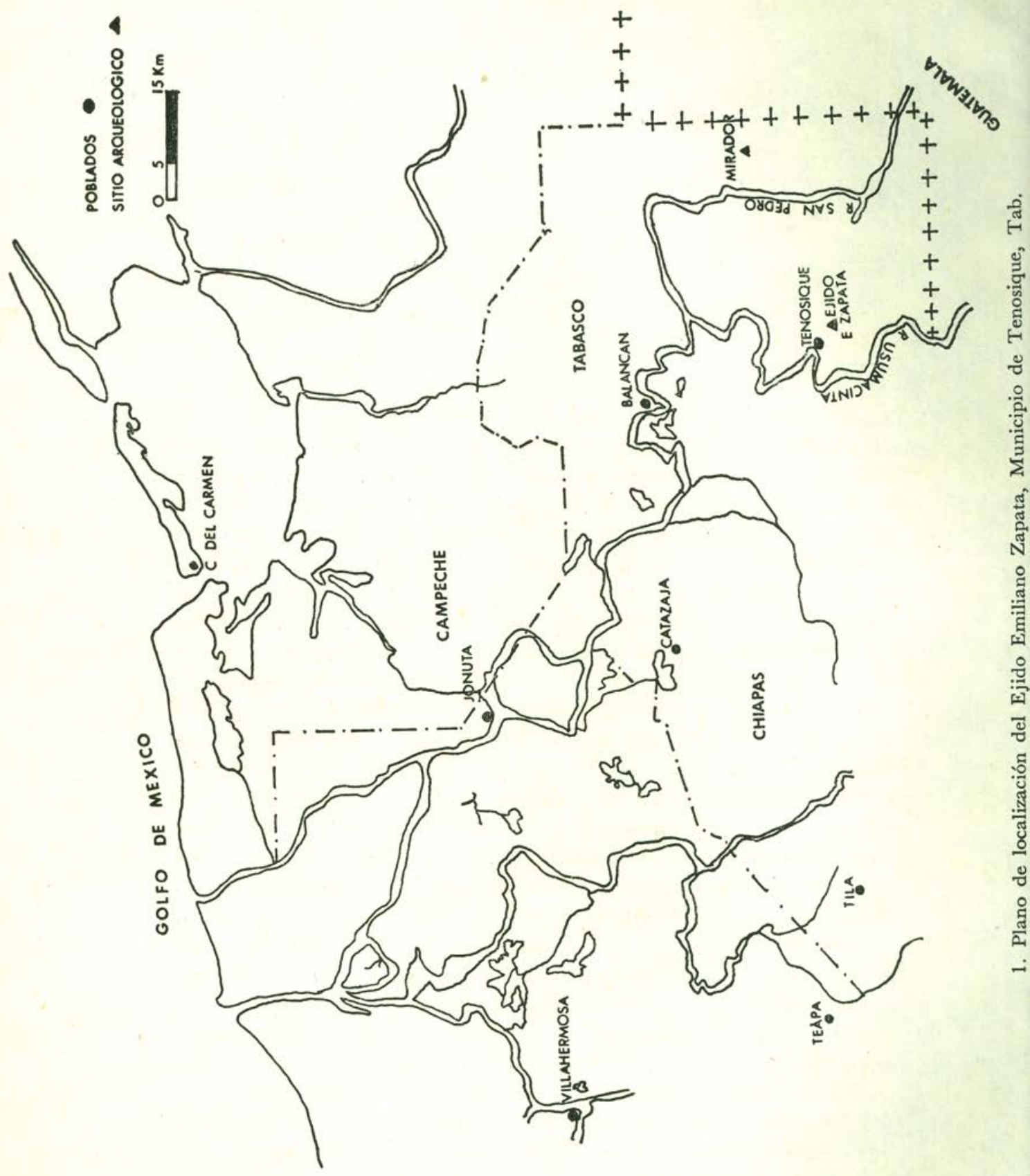


truyen invariablemente el contexto en el que éstos se encontraban, con lo que se invalida la posibilidad de lograr un mejor conocimiento de la problemática que presentaban los grupos que habitaron el actual territorio mexicano.

Aproximadamente a 20 kilómetros al sureste de Tenosique, en el municipio del mismo nombre, Estado de Tabasco, se localiza el ejido Emiliano Zapata, dentro de una zona comprendida entre los ríos Usumacinta y San Pedro Mártir. Esta zona forma parte de la Llanura Costera del Golfo de México donde tuvo su asiento el mayor desarrollo de la cultura olmeca (Piña Chán y Covarrubias; 1964, 5) y también se le ha designado recientemente como Tierras Bajas Noroccidentales del Área Maya (Culbert, 1973, fig. 1; Ochoa y Hernández, 1977), en donde predominaba, antes de la colonización moderna, la selva alta perenifolia. Hoy día, aunque persiste una precipitación pluvial media anual superior a los 2,000 mm. y una temporada de secas muy corta, sólo existen reducidos núcleos de vegetación primaria, predominando los pastizales y cañaverales.

El ejido Emiliano Zapata, al igual que muchos otros de México, cuenta con un sitio arqueológico, y es en la parcela de don José Fonticiella, en la que se localizan varios montículos, donde allá por el mes de marzo de 1978, una gran roca caliza fue removida por un tractor que preparaba la tierra para sembrar caña. El punto preciso de localización se desconoce, ya que por su volumen se depositó en los límites de la parcela, para que no estorbara la actividad de la maquinaria. De este punto fue rescatada por instrucciones del Presidente Municipal, Sr. Emilio Vela Golip, y trasladada a la comandancia donde permanece en depósito.

La roca mide $94 \mathrm{~cm}$. de largo por $88 \mathrm{~cm}$. de ancho y $40 \mathrm{~cm}$. de espesor; afecta la forma de un paralelepípedo rectangular con la particularidad de haber sido labrada con motivos humanos en una de sus caras mayores. La talla se realizó en bajo relieve y grabado. Presenta huellas de erosión por intemperismo, así como las huellas de los aparejos del tractor, como se puede apreciar en la sección inferior izquierda de la escultura.

$\mathrm{Al}$ centro de la cara labrada está un rostro de forma cuadrangular con esquinas redondeadas; la parte superior de la cabeza presenta tres incisiones en forma de "V", siendo la parte central más profunda y corta, mientras que las dos laterales son ligeramente incisas, prolongándose hasta llegar casi a los ojos. Flanquean a la incisión central dos círculos incisos. Los ojos son de forma almendrada y ambos presentan una perforación a manera de iris. De la nariz poco se conserva, pero por los vestigios que de ella quedan da la apariencia de haber 
sido corta y ancha. La boca ocupa la cuarta parte del rostro, es de forma "felinesca", de gruesos labios con las comisuras hacia abajo. E1 labio superior enmarca seis piezas dentales de borde redondeado y sobre el labio inferior se sitúan tres círculos incisos. Abajo de la boca, sobre el mentón, se localizan otros cuatro círculos incisos, distribuidos simétricamente, dos al centro y uno a cada extremo. En ambos lados del rostro, aunque es más claro en el lado derecho por estar menos erosionado y destruido, presenta dos elementos en forma de gancho en un extremo y un círculo en el otro. Los más largos enmarcan la sección de la frente, ojos y parte de la boca, y los cortos parte de la boca y el mentón.

Las orejas, y en este caso también está más claro del lado derecho, se encuentran divididas internamente en dos secciones. En ambos casos existen orejeras realizadas en tres partes: una primera de forma irregular, la segunda circular y la tercera trapezoidal.

En apariencia el rostro antes descrito forma parte de todo un personaje, ya que en la parte inferior de él se representan dos brazos entrecruzados; el pulgar de la mano derecha pasa por entre los dedos índice y medio, sobresaliendo del dorso de la mano en longitud exagerada al resto de ellos, asimismo, el meñique de esta mano está levantado. La mano izquierda, aunque está semidestruida, da la apariencia de tener todos los dedos extendidos con la palma hacia abajo. $\mathrm{Al}$ centro, en el punto donde se entrecruzan los brazos, se encuentran dos elipses concéntricas con una incisión vertical.

Sobre la cabeza se aprecia una delgada barra horizontal unida a otra más gruesa de contorno curvo, con una hendidura en forma de "V" hacia el centro. Apoyados en el conjunto anterior, y sobresaliendo de él, están dos plantas de pies humanos.

Finalmente, en el resto del relieve se distinguen otras líneas o trazos, tanto del lado derecho como del izquierdo.

De lo antes descrito, hemos interpretado esta representación antropomorfa como la de un contorsionista en vista frontal, con el tronco y las extremidades inferiores flexionadas hacia atrás, apoyando los pies sobre la cabeza. Los brazos se representan cruzados al frente y, como elemento sobresaliente sobre ellos, están dos elipses con una incisión vertical, que pensamos es la representación del órgano sexual femenino. El único problema que existiría para poder hacer la interpretación de la posición general del personaje, es el de la colocación de los pies, que en lugar de estar visible el dorso, son las plantas, pero creemos que esto se debe a una libertad del escultor.

En términos generales, el relieve encontrado en Tenosique, Tabasco, guarda cierta semejanza con el monumento 27 de Laguna de Los 


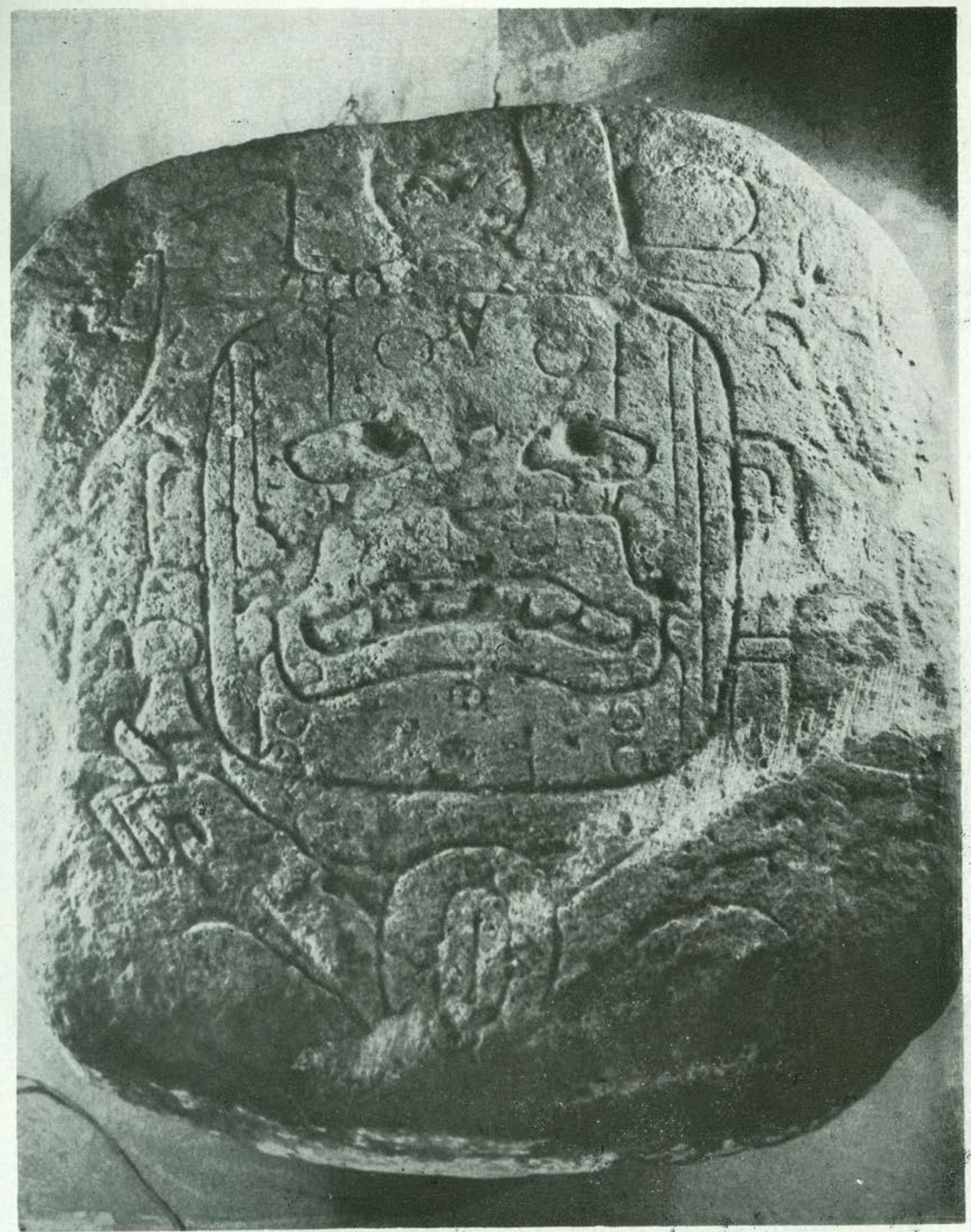

2. Toto tell Relieve de Tenosique, Tab. 

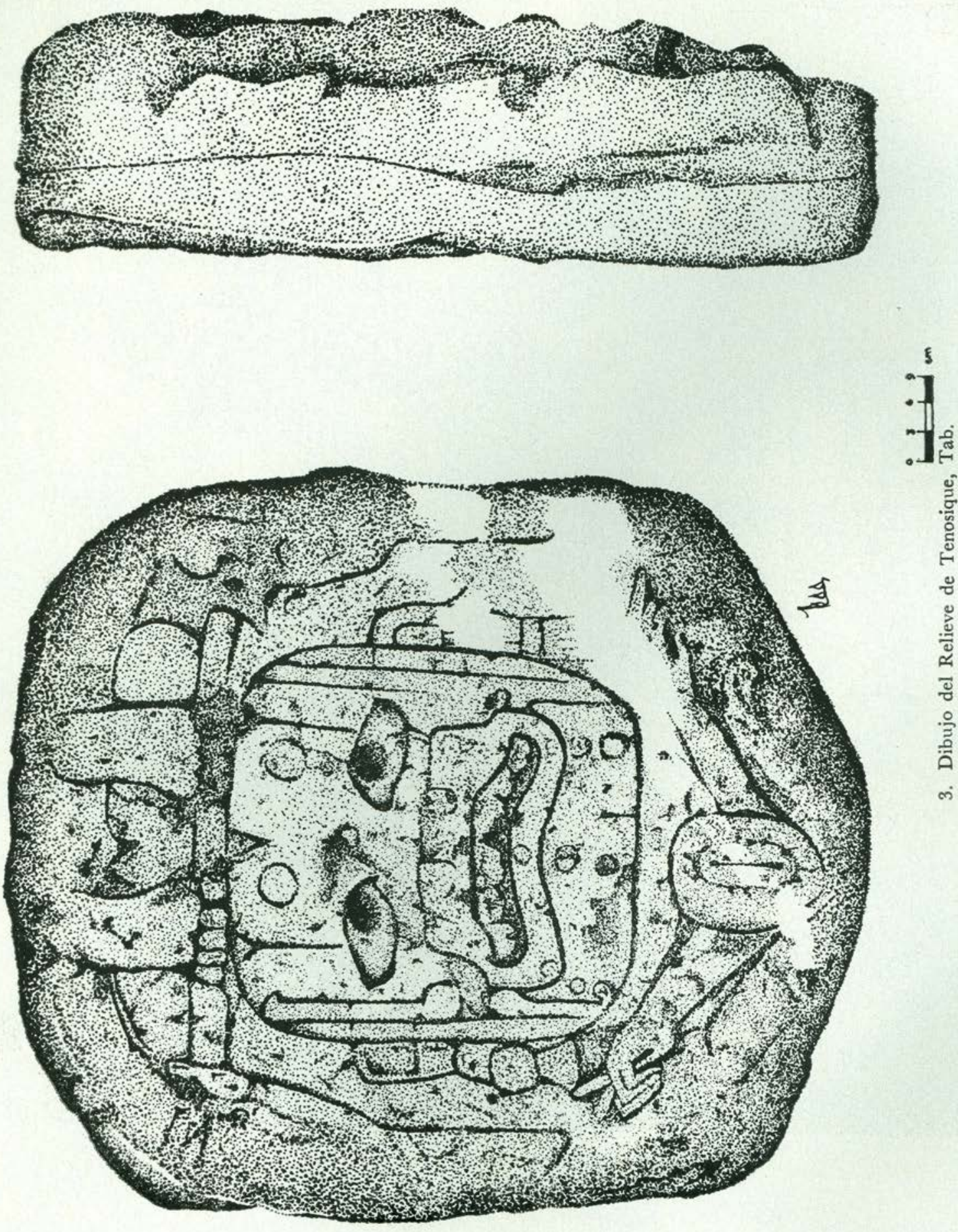


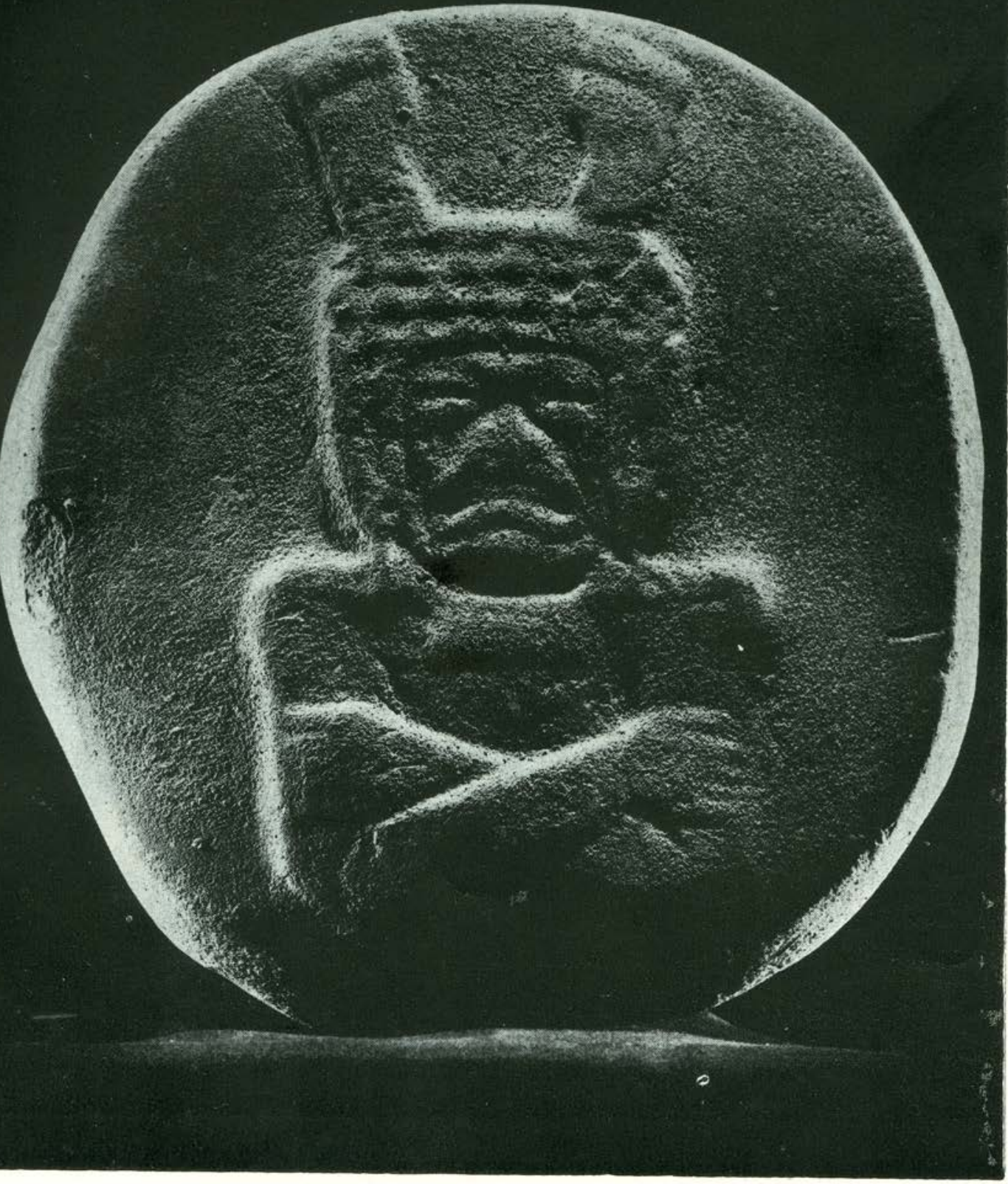

4. Fotografía del Relieve de las Choapas, Veracruz.

Estudios de Cultura Maya. Vol. XII, 1979

Instituto de Investigaciones Filológicas/

Centro de Estudios Mayas, UNAM

http://www.iifilologicas.unam.mx/estculmaya/ 


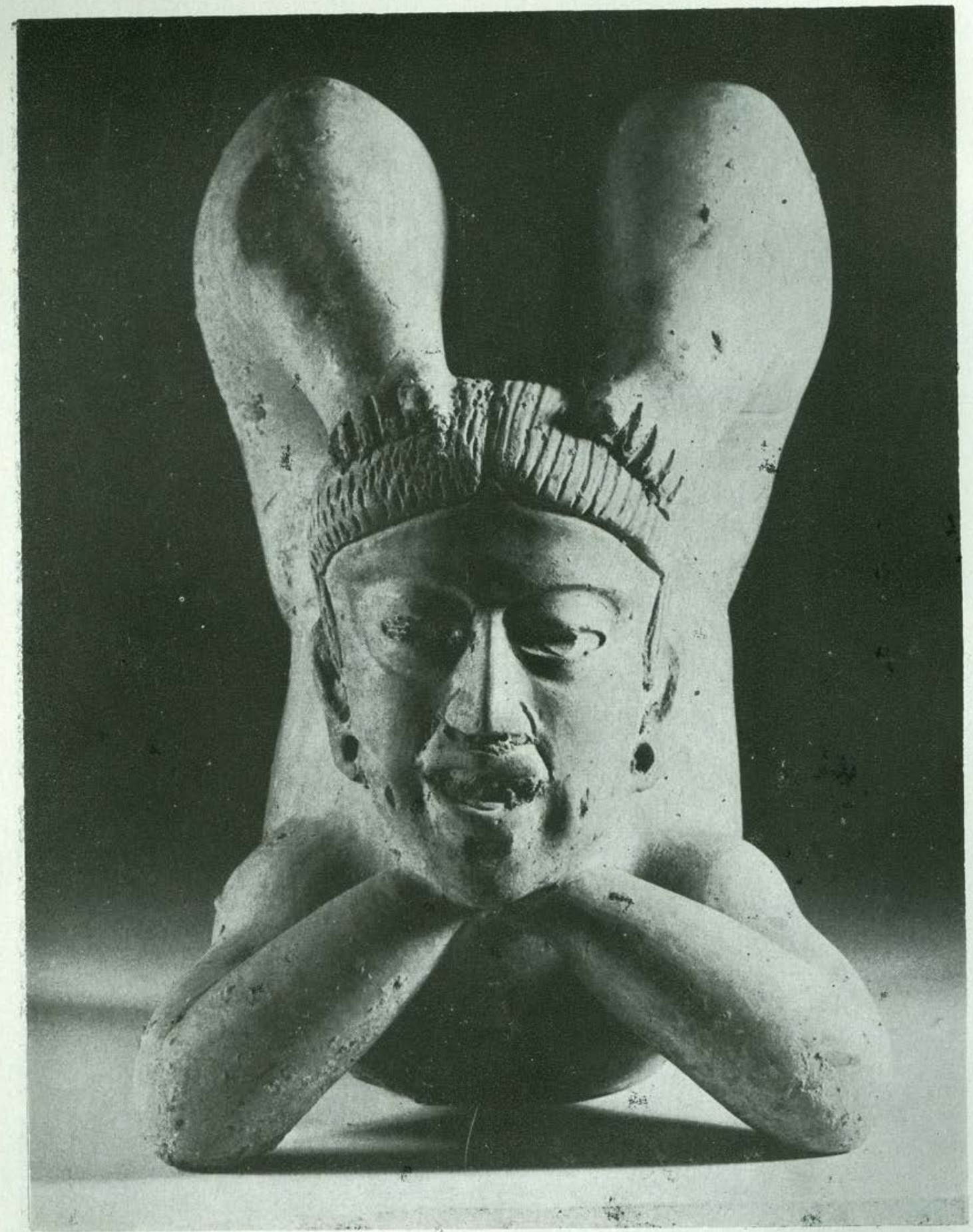

5. Fotografía del Botellón Antropomorfo de Tlatilco, Estado de México. 
Cerros, Ver. (de la Fuente, 1973, 152-153) y con el que se encuentra en el Museo de Santiago Tuxtla, Ver., de procedencia desconocida, clasificado bajo el número 203 del catálogo de Escultura Monumental Olmeca (Ibidem, 257-259). En el sentido particular de la representación de un contorsionista, podría ser similar al caso del monumento número 65 de La Venta, Tab., el cual ha sido descrito como "Extraña representación de una gran cabeza que se apoya sobre brazos desproporcionadamente pequeños"; "Los brazos son como la base que sostiene a la gran cabeza..." (Ibidem, 112); además se encuentra dentro de la descripción la siguiente información: que está completa, muy erosionada, así como que posee acanaladuras en la parte posterior. Estas acanaladuras bien pueden ser el perfil de brazos, tronco y piernas por detrás de la cabeza del personaje.

Otra representación antropomorfa de esta postura corresponde al claro ejemplo del contorsionista de las Choapas, Ver. La escultura, muy erosionada, está ejecutada sobre un bloque de arenisca en forma de disco, de $60 \mathrm{~cm}$. de diámetro por $19 \mathrm{~cm}$. de espesor, realizado el motivo en alto relieve. La figura antropomorfa se sitúa hacia la parte media de una de las caras mayores y tiene los brazos cruzados al frente del pecho por debajo de la cara, apoyando los pies sobre la cabeza. La posición de ellos no es como la que se representa en el bajo relieve de Tenosique, aunque ambos exhiben las plantas de los pies, ésta sí obedece a una realidad anatómica del personaje. Entre otras de las características generales del personaje de las Choapas, está el tocado que porta a manera de casco. La cara es de forma cuadrangular con las esquinas redondeadas; presenta los típicos rasgos olmecas; ceño carnoso, ojos en forma de ranura, nariz corta y ancha, boca "felinesca" y aparentemente sin dientes."

Otros ejemplos de este tipo de posición los tenemos tanto en figurillas como en vasijas antropomorfas procedentes de Tlatilco, Estado de México, relacionadas con la cultura olmeca. La más notable de ellas es la que procede del entierro 154, de la temporada IV (Romano; 1967). Se trata de un botellón antropomorfo realizado en cerámica gris, que sosteniendo la cabeza sobre las manos, gira el cuerpo por detrás, apoyando la planta de los pies en ella. En este caso, la posición de los pies si obedece a una realidad anatómica del personaje, como en el de las Choapas, Ver.; sólo que en el acróbata de Tlatilco, la parte visible de los pies es el dorso y no las plantas.

Elementos particulares que toman parte en esta escultura de Teno-

* Agradecemos al Profr. Arturo Romano y al Museo Nacional de Antropología, la información anterior sobre el relieve de Las Choapas, Ver., dicha pieza se localiza en el Museo bajo el Núm. de inventario 10-162410. 
sique son descritos en el diccionario de motivos y símbolos olmecas de Joralemon (Joralemon; 1971), como a la pieza o monumento de la cultura olmeca a la que se asocian. Así tenemos que las hendiduras en forma de "V" sobre la cabeza, en el citado diccionario aparecen bajo el número 3 , tratándose de uno de los elementos más frecuentes en las representaciones antropomorfas de esta cultura. Como segundo elemento que se encuentra presente en otras esculturas es el ojo almendrado con iris 6-d; la típica boca olmeca, de labios gruesos y comisuras hacia abajo; este elemento aparece bajo el número 19 y es también de los de mayor frecuencia. Los círculos incisos que aparecen distribuidos en el rostro de la escultura que tratamos aparecen en el diccionario bajo los números 91 y 155, en el primer caso sólo uno, y en el segundo se trata de nueve puntos o círculos, con una distribución diferente a la nuestra; en ambos casos han sido interpretados como granos de maíz. Por último, la huella de pie humano aparece bajo el número 178, encontrándose esta representación sólo en el monumento 13 de La Venta, Tabasco; realmente pensamos que no es el caso del monumento de Tenosique.

Tanto la representación de los puntos o círculos, identificados como granos de maíz, así como la representación del órgano sexual femenino, nos lleva a asociar esta escultura con la fertilidad y la agricultura.

Finalmente, este tipo de hallazgo refuerza la existencia de asentamientos olmecas en esta región, con lo cual se fortalece lo planteado por Piña Chán y Covarrubias (1964), ampliándose la presencia no sólo de objetos que son fácilmente transportables como parte de un intercambio o comercio, sino la de escultura monumental (Hernández; 1976) (Ochoa y Hernández, 1977) como es también el monumento de Balancán (El Mirador), así como los trabajos de Ekholm-Miller (1973) en Xoc entre otros muchos. Si bien estos sitios no son de la magnitud de los de La Venta y San Lorenzo que constituyen la zona nuclear de los olmecas (Coe, 1965) (Wicke, 1971) si creemos que es un área que en un futuro puede resultar clave para el entendimiento de lo olmeca en relación a los mayas.

\section{BIBLIOGRAFIA}

Coe, Michael D.

1965 "The Omec Style and its Distribution", en Handbook of Middle American Indians, Vol. 3, 739-775, University of Texas Press.

Culbert, Patrick

1973 The Classic Maya Collapse. University of New Mexico Press, P. Culbert, Ed., Alburquerque. 
DE la Fuente, Beatriz

1973 Escultura Monumental Olmeca, Catálogo. Cuadernos de Historia del Arte No. 1, Instituto de Investigaciones Estéticas, U.N.A.M., México.

Ekholm-Mitler, Susanna

1973 The Olmec Rock Carving at Xoc, Chiapas, México. Papers of the New World Achaeological Foundation, No. 32, Brigham Young University, Provo, Utah.

Hernández Ayala, Martha Ivón

1976 "Una estela olmeca en el área del Usumacinta". Boletín del I.N.A.H., Época II, No. 17, 25-28, México.

Joralemon, Peter D.

1971 A Study of Olmec Iconography. Studies in Pre-Columbian Art and Archaeology, No. 7, Dumbarton Oaks, Trustees for Harvard University, Washington, D. C.

Ochoa, Lorenzo y Martha Ivón Hernández

1977 "Los Olmecas y el Valle del Usumacinta". Anales de Antropología, Vol. XIV, 75-90, U.N.A.M., México.

Piña Chán, Román y Luis Covarrubias

1964 El Pueblo del Jaguar. Consejo para la Planeación del Museo Nacional de Antropología, S.E.P., México.

Romano, Arturo

1967 "Tlatilco". Boletín del I.N.A.H., Época I, No. 30, 38-42, México. Wicke, Charles R.

1971 Olmec and early Art Style in Pre-Columbian Maxico. The University of Arizona Press, Tucson, Arizona. 